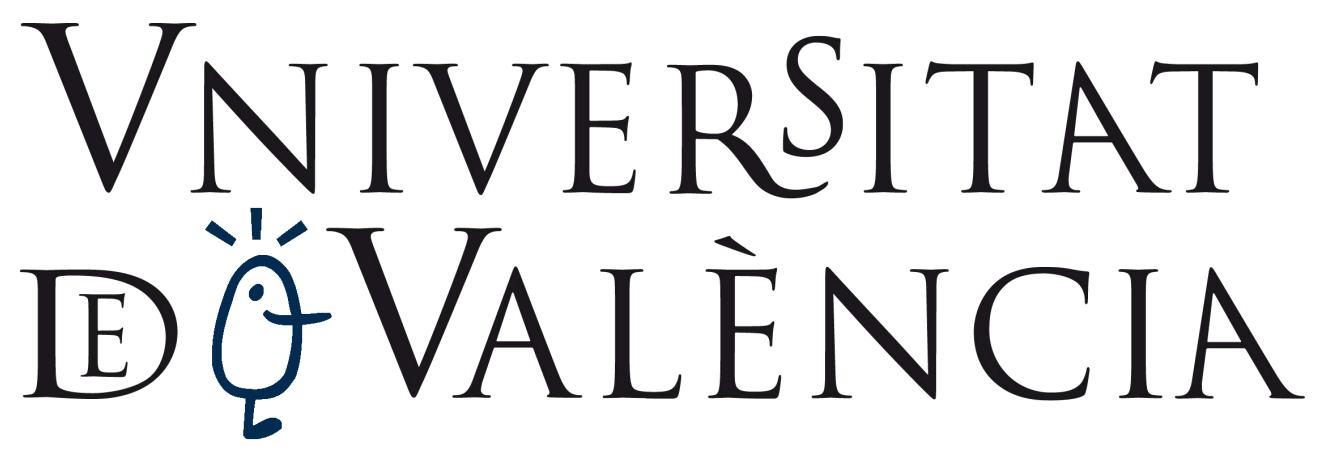

Citation for the original published paper:

Hui ZHANG, Xiao-dong XU, Jing-ya LI, Xiao-feng TAO, Svensson Tommy, Botella Carmen, Performance of power control in inter-cell interference coordination for frequency reuse, The Journal of China Universities of Posts and Telecommunications, Volume 17, Issue 1, 2010, Pages 37-43, ISSN 1005-8885

https://doi.org/10.1016/S1005-8885(09)60421-0

The published paper can be obtained in the following link:

https://www.sciencedirect.com/science/article/pii/S1005888509604210

This is an author's version under creative common license CC-BY-NC-ND 4.0 


\title{
Performance of power control in inter-cell interference coordination for frequency reuse
}

\author{
ZHANG Hui ${ }^{1,2}(\varangle)$, XU Xiao-dong ${ }^{1,2}$, LI Jing-ya ${ }^{1,2}$, \\ TAO Xiao-feng ${ }^{1,2}$, Tommy Svensson ${ }^{3}$, Carmen Botella ${ }^{3}$ \\ 1. Wireless Technology Innovation Institute, Beijing University of Posts and Telecommunications, Beijing 100876, China \\ 2. Key Laboratory of Universal Wireless Communications, Ministry of Education, Beijing University of Posts and Telecommunications, Beijing 100876, China \\ 3. Chalmers University of Technology, Sweden
}

\begin{abstract}
To mitigate inter-cell interference in 3G evolution systems, a novel inter-cell interference coordination scheme called soft fractional frequency reuse is proposed in this article, which enables to improve the data rate in cell-edge. On this basis, an inter-cell power control is presented for the inter-cell interference coordination, and the inter-cell balanced signal to interference plus noise ratio (SINR) among users is established for power allocation, which enables mitigation of inter-cell interference. Especially, the power control is based on a novel exponential kernel equation at higher convergence speed than the traditional arithmetic kernel equations. Numerical results show that the proposed scheme improves the throughput and reduces the blocking rate compared to the existing power control algorithms.
\end{abstract}

Keywords 3G evolution, inter-cell interference, power control, power allocation, exponential iteration

\section{Introduction}

With the continuous growth of the market for wireless communications, there is an increasing need for $3 \mathrm{G}$ evolution systems. For 3GPP organization, the universal mobile telecommunications system (UMTS) is proposed for 3G long term evolution (LTE), which is called as the evolved UMTS terrestrial radio access (UTRA) and UMTS terrestrial radio access network (UTRAN). On the other hand, for 3GPP2 organization, ultra mobile broadband (UMB) is proposed and enhanced, which aims at almost the same requirements as those in 3GPP long term evolution (LTE).

Orthogonal frequency division multiplexing (OFDM) is one of the key technologies for 3G evolution systems. The sub-carriers in OFDM system provide orthogonality for users of the same cell. Hence the intra-cell interference is effectively avoided. However, when the subcarriers are reused among different cells, additional inter-cell interference is

Corresponding author: ZHANG Hui, E-mail: bupt2008@gmail.com introduced in the system.

To mitigate inter-cell interference, three schemes are proposed by 3GPP organization, which respectively are interference randomization, interference cancellation and interference coordination [1]. Among these schemes, the interference coordination approach is the one that receives increasing interest in the framework of 3GPP LTE and 3GPP2 UMB proposals.

As an important method in inter-cell interference coordination, the frequency reuse schemes are researched in plentiful articles. In Refs. [2-3], the frequency reuse factor in cell-center is 1 , while in cell-edge is $1 / 3$. In Ref. [4], it gives a frequency reuse from the point of coloring method in graph theory, which is based on the user equipment (UE). However, its complexity increases as the number of UE increasing. In Ref. [5] an overview is given of contemporary and forward looking inter-cell interference coordination techniques for $4 \mathrm{G}$ OFDM systems, such as adaptive frequency reuse, power control, etc. In Ref. [6] an overview is presented of interference coordination, respectively based on global system knowledge and local system knowledge. In Ref. [7] the ICIC 
gain for the uplink of the 3GPP LTE system is investigated. Moreover, a number of FFR based schemes are compared in Refs. [8-9]. Among these articles, users are divided into cell-center and cell-edge according to the ratio of receiving power from local cell to the co-frequency interfering power from other cells. The frequency reuse factor in cell-center is usually 1 , while in cell-edge is a fraction smaller than 1.

However, when it refers to power allocation for users, many 3GPP proposals share a common power allocation strategy [2-3]. In this case, partial power is assigned to the users in the cell-center, whereas full power is allocated to the users in the cell-edge (this scheme is also referred to as fixed power allocation). However, the performance of these frequency reuse schemes is still significantly lower for the users in the cell-edge area. Moreover, the fixed power allocation scheme may introduce additional interference by allocating full power to users in the cell-edge.

In this scenario, a novel inter-cell coordination scheme called soft fractional frequency reuse (SFFR) is proposed in this article, which has been tested in China FuTURE 4G TDD trials. Moreover, it can effectively improve the data rate in cell-edge. To further mitigate the inter-cell interference, an inter-cell power control algorithm is also considered to dynamically adjust power allocation, and a balanced signal is established to SINR among cells, which enables mitigation of the inter-cell interference.

The remainder of this article is organized as follows: SFFR scheme is introduced in Sect. 2. The inter-cell power control is proposed in Sect. 3. Moreover, the kernel iterative equations in the power control are analyzed, and the process of such inter-cell power control algorithm is introduced. The performance analysis of SFFR and inter-cell power control is made in Sec. 4. Finally, the main conclusions are drawn in Sect. 5.

\section{Soft fractional frequency reuse}

To improve the performance in cell-edge, the SFFR scheme is proposed, which is based on soft frequency reuse (SFR) [3]. As shown in Fig. 1, the characteristics of such reuse schemes are given as follows: the whole cell is divided into two parts, cell-center and cell-edge. In the cell-center, the frequency reuse factor (FRF) is set as 1, while in cell-edge, FRF is dynamic and the frequency allocation is orthogonal with the edge of other cells, which can avoid partial inter-cell interference in cell-edge.

Specially, users in each cell are divided into two major groups according to their geometry factor. In the cell-edge group, users are interference-limited due to the neighboring cells, whereas in cell-center group users are mainly noise-limited. The available frequency resources in the cell-edge are divided into some non-crossing subsets in SFFR.

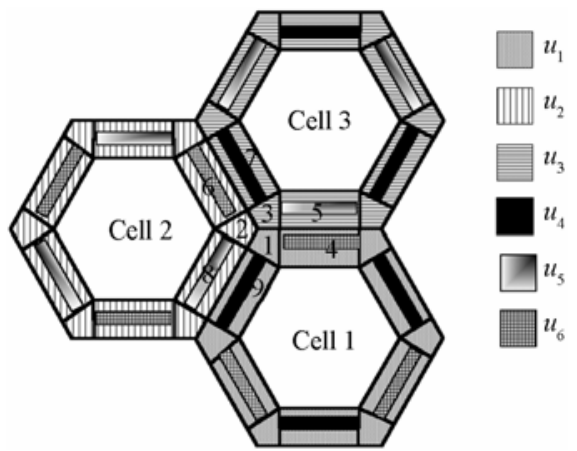

Fig. 1 Concept of soft fractional frequency reuse

The set of available frequency resources in the cell is allocated as follows: the whole frequency band is divided into two disjoint sub-bands, $G$ and $F$, where $G$ is allocated to the cell-center users and $F$ to the cell-edge users. Considering a cluster of 3 cells, as the one shown in Fig. 1, let $F=F_{1} U$ $F_{2} \cup F_{3}$, where $F_{i}$ denotes the subset of frequencies allocated to cell $i, \quad(i=1,2,3)$, and the subsets $F_{i}$ may be overlapped with each other.

Since the cell-edge users are easily subject to co-frequency interference, the frequency assignments to the cell-edge users greatly rely on radio link performance and system throughput. Generally, the cell-edge can be divided into 12 regions, as the ones marked by 1, 4, and 9 in cell 1 (see Fig. 1). Therefore, in a cluster of 3 adjacent cells, there are 9 parts in the cell-edge corner, which are in the shaded area. Moreover, this SFFR model is considered as an example to deduce the design of the available frequency band assignment for the fields marked by $1,2, . ., 9$.

In SFFR, all the available frequencies in cell-edge are divided into 6 non-overlapping subsets. Such subsets are respectively $u_{1}, u_{2}, u_{3}, u_{4}, u_{5}$ and $u_{6}$, while the subset in cell-center is $u_{0}$. First, the authors select frequency from the subsets $u_{1}, u_{2}, u_{3}$. If it is insufficient, choose frequency from $u_{4}, u_{5}, u_{6}$. If inter-cell interference increases, add frequency into $u_{4}, u_{5}, u_{6}$, and decrease the cover area in cell-edge. If such interference is controlled in a low extension, decrease the frequency in subsets of $u_{4}, u_{5}, u_{6}$, and increase the cover area in cell-edge, which enables improvement in the frequency utilization. Moreover, the authors assume $A_{1 / 3}=$ $\left\{u_{1}, u_{2}, u_{3}\right\}, A_{2 / 3}=\left\{u_{4}, u_{5}, u_{6}\right\}$ and $A_{3 / 3}=\left\{u_{0}\right\}$, where $A_{1 / 3}$ 
denotes the frequency set with $1 / 3$ reuse, $A_{2 / 3}$ denotes the frequency set with $2 / 3$ reuse and $A_{3 / 3}$ denotes the frequency set with FRF equals to 1.

Following the definition of Ref. [4], the FRF is defined for the SFFR scheme. Hence, the FRF can be obtained as follows:

$$
\eta=\frac{\frac{1}{3}\left|A_{1 / 3}\right|+\frac{2}{3}\left|A_{2 / 3}\right|+\frac{3}{3}\left|A_{3 / 3}\right|}{\left|A_{1 / 3}\right|+\left|A_{2 / 3}\right|+\left|A_{3 / 3}\right|}
$$

where the symbol $|\cdot|$ stands for the cardinality of frequency set. By considering $|A|=\left|A_{1 / 3}\right|+\left|A_{2 / 3}\right|+\left|A_{3 / 3}\right|$, the following relation is obtained:

$|A|=\left|u_{0}\right|+3\left|u_{1}\right|+3\left|u_{4}\right|$

Combining Eqs. (1) and (2), the FRF is computed as:

$\eta=\frac{\left|u_{0}\right|}{|A|}+\frac{1}{3} \times \frac{\left|u_{1}\right|}{|A|} \times 3+\frac{2}{3} \times \frac{\left|u_{2}\right|}{|A|} \times 3$

From Eq. (2), one can get the equation about $u_{1}$ as follows:

$$
\left|u_{1}\right|=\frac{|A|-3 \times\left|u_{4}\right|-\left|u_{0}\right|}{3}
$$

Following the example of cell 1 , the number of available frequencies in cell-center is $\left|u_{0}\right|$, whereas in the cell-edge is $\left|u_{1}\right|+2\left|u_{4}\right|$. Assume that $\left|u_{0}\right|=k\left(\left|u_{1}\right|+2\left|u_{4}\right|\right)$, where $k$ is a constant parameter, then $\left|u_{4}\right|$ can be obtained from Eq. (4):

$\left|u_{4}\right|=\frac{\left|u_{0}\right|}{3 k}-\frac{\left|u_{0}\right|}{9}-\frac{|A|}{4}$

Finally, taking into account Eqs. (4) and (5), Eq. (3) can be expressed in terms of $\left|u_{0}\right|$ :

$\eta=\frac{1}{12}+\left(\frac{1}{3 k}+\frac{5}{9}\right) \frac{\left|u_{0}\right|}{|A|}$

It can be seen from Eq. (6) that as FRF grows, the available frequency resources in cell-center increase, while those in cell-edge decrease.

However, the SFFR scheme, as many other 3GPP LTE proposals, allocates partial power to users in cell-center and full-power to users in cell-edge, which may result in additional interference. To mitigate the inter-cell interference, an inter-cell power control algorithm is proposed in the next section for optimizing the power allocation.

\section{Inter-cell power control}

Intra-cell power control is an important technology in CDMA systems, since it reduces the distance effect and mitigates intra-cell interference among users. However, in
OFDM systems, intra-cell sub-carriers are orthogonal, and thus the intra-cell interference is effectively avoided. In this case, the inter-cell interference becomes the major source of interference.

In this section, an inter-cell power control algorithm is presented to adjust the power allocation in sub-carriers in OFDM systems. Specially, an exponential kernel iterative equation is also proposed for the inter-cell power control, whose performance is better than traditional arithmetic equations. By means of inter-cell power control, the balanced SINR can be established among cells, which not only reduces inter-cell interference, but also improves system performance.

\subsection{Kernel iterative equation}

For power control algorithm, the kernel iterative equation is an important factor, which directly affects the performance of each algorithm. In this article, a definition is given. If SINR in kernel equation is in the form of arithmetic operations, such as multiplication, division, addition and subtraction, the authors classify it as arithmetic kernel equation. At the same time, if SINR in kernel equation is in the form of exponential operations, it will be classified as exponential kernel equation.

In traditional power control algorithms, most of kernel equations are arithmetic kernel equations, such as DB and DPC. Also for arithmetic kernel equation, its convergence speed is usually slowly in iterations. As classical arithmetic kernel equations, the kernel equations of DB and DPC are written as follows [10]:

$p^{(n+1)}=\left\{\begin{array}{l}\left(1+\frac{\gamma^{\text {th }}}{\gamma^{(n)}}\right) p^{(n)} ; \mathrm{DB} \\ p^{(n+1)}=p^{(n)} \frac{\gamma^{\text {th }}}{\gamma^{(n)}} ; \text { DPC }\end{array}\right.$

As shown in Eq. (7), $\gamma^{\text {th }}$ is the threshold SINR, $\gamma^{(n)}$ is the SINR in the $n^{\text {th }}$ iteration and $p^{(n)}$ is the power in the $n^{\text {th }}$ iteration. It can be seen that SINR is in a form of fraction, where the threshold SINR is divided by current iterative SINR. Except for DB and DPC, many other kernel equations are evolved from such fractional SINR, but only modified with several extra parameters.

To improve the performance of the kernel iterative equation, an exponential kernel equation is proposed, whose convergence speed is faster than traditional arithmetic kernel equations. On the other hand, the previous iterative power is added as a weighted feedback in kernel equation. The proposed exponential iterative equation can be expressed as follows: 
$p^{(n+1)}=\mu \mathrm{e}^{k\left(\gamma^{\mathrm{h}}-\gamma^{(n)}\right)} p^{(n)}+(1-\mu) p^{(n-1)}$

where $k$ is a positive constant, $\mu$ is a relaxation factor $(0<\mu \leqslant 1)$ and $p^{(n-1)}$ is the power in the $(n-1)$ th iteration. The term related to the $p^{(n-1)}$ power can be seen as a weighted feedback, which helps maintain the robustness in iteration.

To make SINR converge into a fixed value in iteration, the kernel iterative equation needs to meet several requirements, namely, nonnegativity, monotonicity and scalability [11]. Furthermore, the proof for such exponential kernel iterative equation is given as follows.

1) Nonnegativity

Assume that $p^{(n)} \geqslant 0$ and $p^{(n-1)} \geqslant 0$. Hence, from Eq. (8), one can obtain $p^{(n+1)} \geqslant 0$, which means such kernel equation is nonnegative.

2) Monotonicity

If $p^{(n+1)}>p^{(n)}$ and $p^{(n)}>p^{(n-1)}$, the following can be obtained:

$p^{(n+2)}-p^{(n+1)}=\mu \mathrm{e}^{k\left(\gamma^{\text {th }}-\gamma^{(n)}\right)}\left(p^{(n+1)}-p^{(n)}\right)+(1-\mu)\left(p^{(n)}-p^{(n-1)}\right)$

Combined with the known conditions, one can get the following inequality:

$\mu \mathrm{e}^{k\left(\gamma^{\mathrm{T}}-\gamma^{(n)}\right)}\left(p^{(n+1)}-p^{(n)}\right)>0$

$(1-\mu)\left(p^{(n)}-p^{(n-1)}\right)>0$

Finally, considering Eqs. (10) and (11), one can get $p^{(n+2)}>p^{(n+1)}$ in Eq. (9), which proves the monotonicity of the exponential kernel equation.

3) Scalability

Let $\alpha$ denote any real coefficient. Then, if $\alpha$ multiplies such kernel equation on the left, the following can be obtained:

$$
\begin{aligned}
\alpha p^{(n+1)}= & \alpha\left[\mu \mathrm{e}^{k\left(\gamma^{\mathrm{h}}-\gamma^{(n)}\right)} p^{(n)}+(1-\mu) p^{(n-1)}\right]= \\
& \mu \mathrm{e}^{k\left(\gamma^{\text {th }}-\gamma^{(n)}\right)} \alpha p^{(n)}+(1-\mu) \alpha p^{(n-1)}
\end{aligned}
$$

It can be concluded from the simplified process in Eq. (12) that such kernel equation is scalability.

The above proof proves that the proposed kernel equation is nonnegativity, monotonicity and scalability. Therefore, it can be considered that such equation can be converged into a fixed value in iteration.

\subsection{Power control algorithm}

In inter-cell power control, the principle of inter-cell balanced SINR among cells is used for mitigating inter-cell interference. By means of inter-cell power control, a balanced
SINR can be established for different users among inter-cells, which helps mitigate inter-cell interference and improve system performance. This algorithm is introduced as follows.

In the initial state, users in intra-cell are divided into cell-center users and cell-edge users according to the power ratio (PR). Specifically, the PR is defined as the ratio of useful power and co-frequency interference from other cells. If the PR is higher than a standard value, such user is classified into cell-center user. Otherwise, such user is classified into cell-edge user. To mitigate the interference from cell-center users, these users are allocated with lower power. However, for those cell-edge users, they are allocated with higher power, which can improve the performance under poor channel conditions.

On the other hand, the threshold SINR can be set for different users and class of services. According to the requirements of quality of service (QoS), four basic service types are defined by 3GPP, which are conversational, streaming, interactive and background, respectively. In addition, if perfect channel state information (CSI) is available at the transmitter, the expected SINR can be higher. Otherwise, the expectation for SINR can be lower. Combined with service types and CSI, it can get the order of priorities for SINR. Therefore, the threshold SINR is defined by each user taking into account the different service type and available CSI. These values are optimal and can be dynamically modified to follow a change in the service type.

Once the initial parameters are established, the inter-cell power control algorithm starts the power adjustment for achieving the required threshold SINR in the inter-cell users. Taking a single co-frequency sub-carrier as an example, the steps of this iterative algorithm are as follows:

Step 1 Set the initial parameters, such as the initial power $p_{i j}^{(0)}$ from cell $j$ to user $i$, the threshold SINR $\gamma_{i}^{\text {th }}$, and the noise power $v_{i}$.

Step 2 Compute the first power $p_{i i}^{(1)}$ :

$p_{i i}^{(1)}=p_{i i}^{(0)} \frac{\gamma_{i}^{\text {th }}}{\gamma_{i}^{(0)}}$

Step 3 Compute $\gamma_{i}^{(1)}$ when the power is $p_{i i}^{(1)}$

$\gamma_{i}^{(1)}=\frac{g_{i i} p_{i i}^{(1)}}{\sum_{j=1, j \neq i}^{N} g_{i j} p_{i j}^{(1)}+v_{i}}$

Step 4 According to the following kernel iterative equation, compute the modified power $p_{i i}^{(n+1)}$ in the next iteration $(n \geqslant 1)$ : 


$$
p_{i i}^{(n+1)}=\mu \mathrm{e}^{k\left(\gamma_{i}^{\mathrm{h}}-\gamma_{i}^{(n)}\right)} p_{i i}^{(n)}+(1-\mu) p_{i i}^{(n-1)}
$$

Step 5 Compute $\gamma_{i}^{(n+1)}$ when the power is $p_{i i}^{(n+1)}$ :

$$
\gamma_{i}^{(n+1)}=\frac{g_{i i} p_{i i}^{(n+1)}}{\sum_{j=1, j \neq i}^{N} g_{i j} p_{i j}^{(n+1)}+v_{i}}
$$

Step 6 If $\left|\gamma_{i}^{(n+1)}-\gamma_{i}^{\text {th }}\right| \leqslant \varepsilon$, the algorithm stops and the optimal power allocation is $p_{i i}^{(n+1)}$. Else, go to the next step.

Step 7 If $p_{i i}^{(n+1)} \leqslant p_{\max }, n=n+1$, go back to step 4. If not, the user with minimum SINR requirement is removed and the priorities are reset. Then, the algorithm goes back to step 1.

In the first step, on the basis of the initial parameters, the first iterative power is computed by the arithmetic kernel equation, and the first SINR under such power can be obtained by the SINR equation. On this basis, the next iterative power is updated by the exponential kernel equation, where the first power is as the feedback power, and the first SINR is as a variable. Then, the new SINR under the iterative power can be obtained by SINR equation. If such SINR approximates to the threshold value, stop iteration and output the power; otherwise, go back to continue the iterative process, until current SINR approximates to the threshold value.

Because the maximum power is constrained in power allocation, if the iterative power is beyond the scope of the maximum power, still the current SINR doesn't reach the threshold value, remove such user, and reset the initial parameters and priorities for users. Then start the new iterative process again.

\section{Performance analysis}

To compare the performance of the SFFR scheme and the inter-cell power control method, simulations are carried out. The simulation parameters are referred from 3GPP LTE proposals [12]. Consider 27 cells, with a cell radius of $1 \mathrm{~km}$, all users are uniformly distributed in the cell area and the wraparound technique is invoked. Furthermore, the center carrier frequency is assumed to be $2 \mathrm{GHz}$, the system bandwidth is set at $10 \mathrm{MHz}$ with the bandwidth of each subcarrier equal to $15 \mathrm{kHz}$, the number of subcarriers is 600 and the overall transmit power per cell equal to $43 \mathrm{dBm}$. Moreover, the path loss model is modeled as $P(d)=128.1+37.6 \lg d$

First, the authors compare the performance of SFR and SFFR. Then, the convergence speed of different kernel iterative equations is analyzed. Moreover, based on the SFFR scheme, the throughput and the block loss rate are compared, which are respectively taken by the fixed power allocation, DB algorithm, DPC algorithm and the proposed power control algorithm.

Fig. 2 compares the average data rate in cell-edge for SFR and SFFR, where the FRF is set as 8/9. It can be seen that the average data rate in cell-edge decreases as the number of users per cell increases. However, the SFFR scheme outperforms the SFR scheme for a given number of users per cell. Specially, as the increase of users, the improvement by the SFFR scheme is more than the SFR scheme, which shows that it is more effective when the number of users is large.

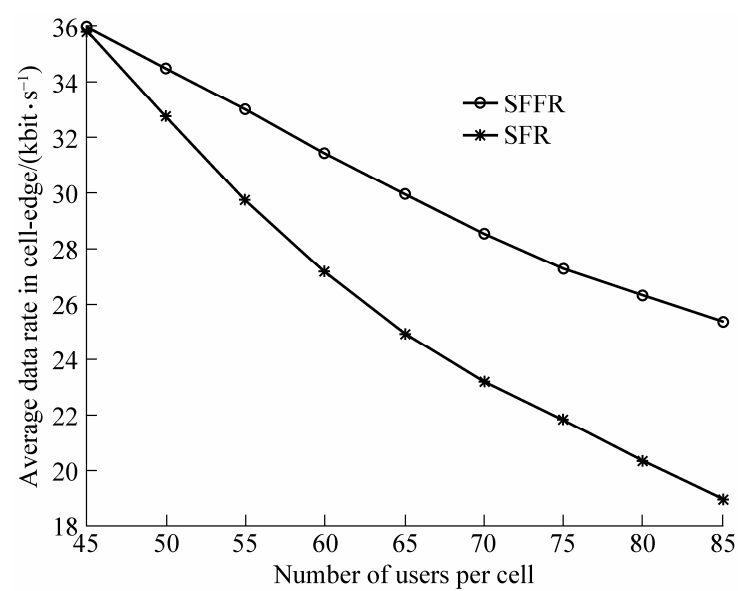

Fig. 2 Comparison of average data rate in cell-edge

Fig. 3 shows the convergence of the DB, DPC and exponential kernel equations. It can be seen that it is a gradual convergence process for DB and DPC equations, which reaches to the threshold SINR in the end. For the proposed equation, however, its iterative speed is faster than DB and DPC. Furthermore, the reason is that DB and DPC are in a form of arithmetic equations, which is a slowly convergence process compared with exponential equation, and the result is shown in Fig. 3. On the other hand, it can be seen that the robustness of the proposed equation is better than DB and DPC, which shows the weighted feedback in the proposed equation effectively improves the stability of iterative convergence. However, for DB and DPC, there are some vibrations in this process, especially when the difference of threshold SINR and the initial SINR is large.

In Fig. 4, the authors compare the throughput in cell-center when four methods are taken at different FRF values. As shown in Fig. 4, with the increase of FRF, the average throughput gradually increases in the cell-center. The reason is that users in cell-center are allocated with more sub-carriers 
when the FRF is large, which improves the average throughput for good channel conditions in the cell-center. When the FRF is small, users in the cell-center are allocated with fewer sub-carriers, while with more sub-carriers in the cell-edge, which decreases the average throughput for poor channel conditions in the cell-edge.

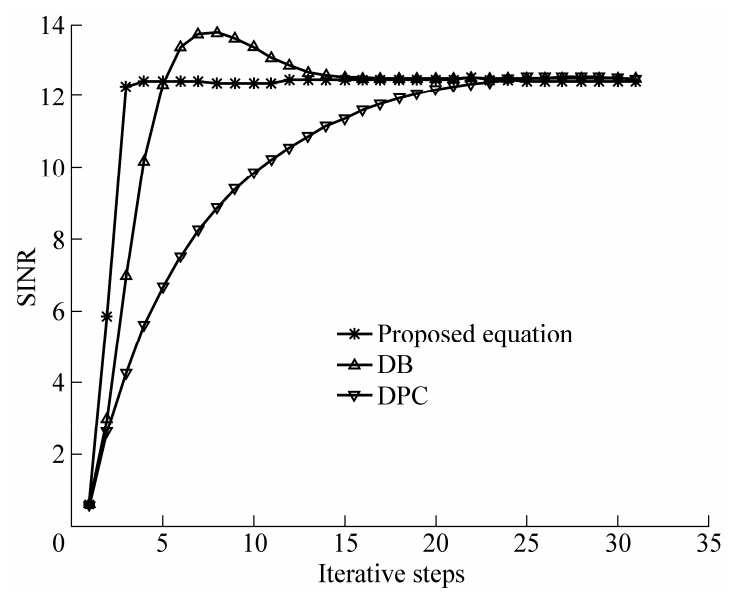

Fig. 3 Convergence of kernel iterative equations

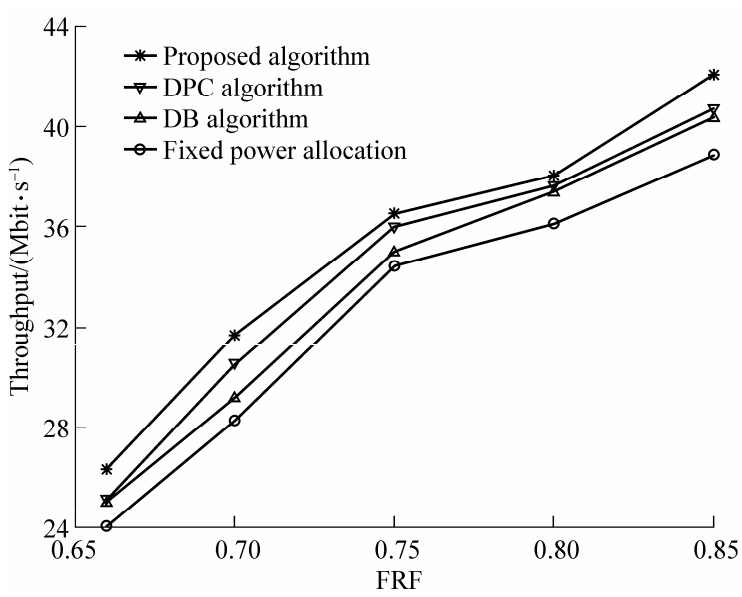

Fig. 4 Comparison of throughput in cell-centre

Moreover, Fig. 4 shows that the proposed exponential kernel equation outperforms the fixed power allocation, DB and DPC algorithms. The fixed power allocation method assigns partial power to users in cell-center and full power to users in the cell-edge. However, for the proposed power control method, it establishes a principle of inter-cell balanced SINR, which dynamically changes the power by iterations to make SINR to the threshold value. Generally, the threshold SINR is an optimum value for users, which not only meets the requirements for different services, but also reduces extra power to interfere with co-frequency users in other cells. Naturally, the average throughput is improved by such threshold SINR .
Finally, Fig. 5 shows the blocking rate in cell-edge. It can be seen that for users in cell-edge, the blocking rate is reduced more after using power control method. From this result, it illustrates that the interference brought by power allocation is one of important reasons for the blocking rate increases in fixed power allocation method.

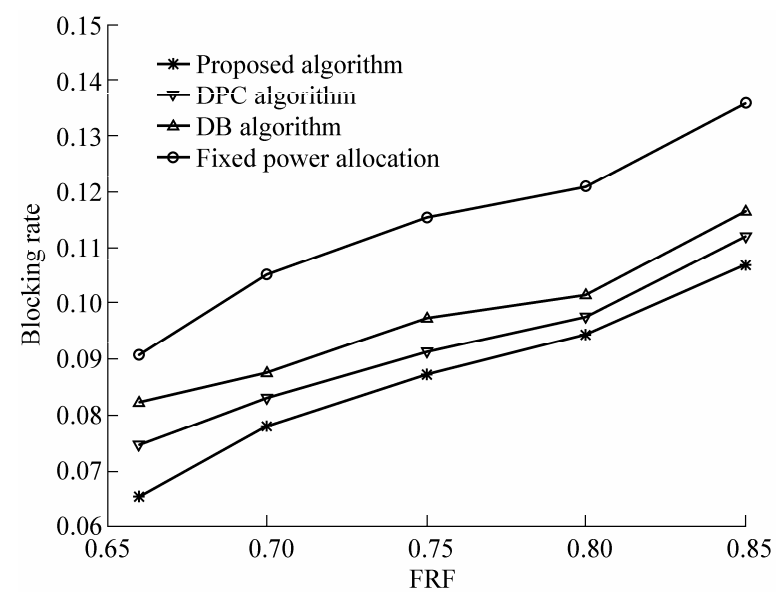

Fig. 5 Comparison of blocking rate in the cell-edge

Moreover, in the fixed power allocation, full power is allocated to users in the cell-edge. This power allocation improves the partial users' performance, while it also introduces additional interference to co-frequency users in other cells. When the number of users in cell-edge increases, this interference increases, and hence the blocking rate is growing. On the other hand, when a dynamical power control method is performed and a balanced SINR among users is established, the additional interference introduced by the fixed power allocation can be effectively avoided. Therefore, the blocking rate is significantly reduced when the DB, DPC and the proposed exponential kernel equation are simulated.

\section{Conclusions}

To mitigate inter-cell interference, a novel inter-cell interference coordination scheme called SFFR is proposed in this article, which can effectively improve the data rate in cell-edge. The numerical results show that compared with the SFR scheme, the SFFR scheme improves the performance in the cell-edge.

On this basis, an inter-cell power control algorithm is proposed, which is based on a novel exponential kernel iterative equation and the principle of inter-cell balanced SINR. Furthermore, the performance of the proposed power control algorithm outperforms the fixed power allocation, DB algorithm and DPC algorithm. Specially, the convergence 
speed of the proposed kernel equation is faster than traditional arithmetic kernel equations with the same iterative steps. By means of this algorithm, it enables improvement in the throughput and reduces the blocking rate.

\section{Acknowledgements}

This work was supported by Sino-Swedish Project (2008DFA12110), Key Project of BMSTC (D08080100620802), the National Natural Science Foundation of China (60872048), and National Science and Technology Special Project 'Group Cell' (2009ZX03003-011).

\section{References}

1. 3GPP TR 25.912. Feasibility study for evolved UTRA and UTRAN. 2007

2. 3GPP R1-050738. Interference mitigation considerations and results on frequency reuse. 2005

3. 3GPP R1-050507. Soft frequency reuse scheme for UTRAN LTE. 2005

4. 3GPP R1-050896. Description and simulations of interference management technique for OFDMA based E-UTRA downlink evaluation. 2005

5. Boudreau G, Panicker J, Guo N, et al. Interference coordination and cancellation for 4G networks. IEEE Communications Magazine, 2009, 47(4): 74-81

6. Necker M C. Interference coordination in cellular OFDMA networks. IEEE Network, 2008, 22(6): 12-19

7. Racz A, Reider N, Fodor G. On the impact of inter-cell interference in LTE. Proceedings of IEEE Global Telecommunications Conference (GLOBECOM’08), Nov 30-Dec 4, 2008, New Orleans, LA, USA. Piscataway, NJ, USA: IEEE, 2008: 6p

8. Simonsson A. Frequency reuse and intercell interference co-ordination in E-UTRA. Proceedings of the 65rd Vehicular Technology Conference (VTC-Spring'07), Apr 22-25, 2007, Dublin, Iseland. Piscataway, NJ, USA: IEEE, 2007: 3091-3095

9. Necker M C. Local interference coordination in cellular 802.16e networks. Proceedings of the 66th Vehicular Technology Conference (VTC-Fall'07): Vol 2, Sep 30-Oct 3, Baltimore, MD, USA. Piscataway, NJ, USA: IEEE, 2007: 1741-1746

10. Foschini G J, Miljanic Z. A simple distributed autonomous power control algorithm and its convergence. IEEE Transactions on Vehicular Technology, 1993, 42(4): 641-646

11. Yates R D. A framework for uplink power control in cellular radio systems. IEEE Journal on Selected Areas in Communications, 1995 13(7): 1341-1347

12. 3GPP TR25.814. Physical layer aspects for evolved UTRA. 2006 